TRANSACTIONS OF THE

AMERICAN MATHEMATICAL SOCIETY

Volume 358, Number 4 , Pages 1827-1846

S 0002-9947(05)03803-1

Article electronically published on October 31, 2005

\title{
ON THE HYPERBOLICITY OF THE PERIOD-DOUBLING FIXED POINT
}

\author{
DANIEL SMANIA
}

\begin{abstract}
We give a new proof of the hyperbolicity of the fixed point for the period-doubling renormalization operator using the local dynamics near a semi-attractive fixed point (in a Banach space) and the theory of holomorphic motions. We also give a new proof of the exponential contraction of the Feigenbaum renormalization operator in the hybrid class of the period-doubling fixed point: our proof uses the non-existence of invariant line fields in the perioddoubling tower (C. McMullen), the topological convergence (D. Sullivan), and a new infinitesimal argument.
\end{abstract}

\section{Introduction AND STATEMENT OF RESUlts}

A unimodal map is an even (real-analytic) map $f:[-1,1] \rightarrow[-1,1]$, with $f(-1)=f(1)=1$, so that 0 is its unique critical point, which is quadratic. A unimodal map is period-doubling renormalizable if there exists an interval $[\rho,-\rho] \subset$ $[-1,1]$ satisfying:

i. $f^{2}(\rho)=f^{2}(-\rho)=\rho$,

ii. $f^{2}([\rho,-\rho]) \subset[\rho,-\rho]$,

iii. 0 is the unique critical point for $f^{2}$ in $[\rho,-\rho]$.

Define the period-doubling renormalization of $f$ as the unimodal map

$$
\mathcal{R} f(z):=\frac{1}{\rho} f^{2}(\rho z) .
$$

Roughly speaking, one of the main objectives of renormalization theory is to study the behavior of operators like the period-doubling renormalization operator.

The renormalization theory in one-dimensional dynamics had its origin in the observation of universality in families of unimodal maps by Feigenbaum and CoulletTresser. They conjectured that such universality could be explained by the existence of a hyperbolic fixed point (the so-called period-doubling fixed point) for the period-doubling renormalization operator (defined in the "space of unimodal maps") and the characterization of its stable manifold. Lanford [Lan] proved existence of such fixed point, and Sullivan $[\mathrm{Su}$ introduced quasiconformal methods in renormalization theory and proved uniqueness of such a fixed point. He also proved that the renormalizations of maps in the so-called hybrid class (see the definition

Received by the editors March 19, 2003 and, in revised form, July 16, 2004.

2000 Mathematics Subject Classification. Primary 37F25, 37E20; Secondary 37F45.

Key words and phrases. Renormalization, parabolic domain, petals, holomorphic motion, Feigenbaum, period-doubling, universality, semi-attractive, hyperbolicity.

This work was partially supported by CNPq-Brazil grant 200764/01-2, University of Toronto and USP-São Carlos.

(C)2005 American Mathematical Society Reverts to public domain 28 years from publication 1827 
in Section 2.2) of the period-doubling fixed point converges to this fixed point. McMullen [McM96] proved that in the hybrid class of the period-doubling fixed point the convergence to the period-doubling fixed point is indeed exponentially fast.

Finally, Lyubich Lyu99 proved the Feigenbaum-Coullet-Tresser conjecture asserting that the period-doubling fixed point is hyperbolic, with a codimension one stable manifold. Furthermore, Lyubich proved that the hybrid class of the perioddoubling fixed point studied by Sullivan and McMullen coincides with the stable manifold of this fixed point. The results cited above make deep use of methods of complex dynamics and represent a sample of the importance of such methods in the development of one-dimensional dynamics in the last two decades. The main goal of this work is to provide a simpler and shorter proof to part of McMullen and Lyubich's results in [McM96] and Lyu99, in order to make these results more accessible to a wider audience.

We will provide a new approach to the following result:

Main Theorem ([Lyu99; see also [Lan, [Su, [McM96]). The period-doubling renormalization operator restricts to a compact operator on an open set of a Banach space $\mathcal{B}$ of holomorphic functions. This operator has a hyperbolic fixed point $f^{*}$, with codimension one stable manifold. Renormalizations of any infinitely renormalizable real-analytic unimodal map eventually belong to the stable manifold of $f^{*}$ in $\mathcal{B}$.

We will postpone the precise definitions and statements until Section 2.2, The reader will observe that we assume the period-doubling combinatorics just to simplify the notation: the argument in the proof of the Main Theorem works as well to prove the hyperbolicity of real periodic points of the renormalization operator.

\section{Preliminaries AND PRecise Statements}

2.1. Notation. For a subset $V \subset \mathbb{C}$ and $\lambda \in \mathbb{C}$, denote $\lambda V:=\{\lambda x: x \in V\}$. If $f$ is a holomorphic function, we will use the notation $\left.f^{-1} V\right|_{0}$ for the connected component of $\{x: f(x) \in V\}$ which contains 0 . If $x_{n}$ converges to $x$ when $n$ goes to infinity, we will write $x_{n} \rightarrow_{n} x$ or simply $x_{n} \rightarrow x$. In this work we will deal with a smooth operator $\mathcal{R}$, whose domain is in a (affine) Banach space of functions. To avoid confusion, we will denote by $D \mathcal{R}_{f}$ the derivative of the operator $\mathcal{R}$ at $f$, and by $D(\mathcal{R} f)$ the derivative of the function $\mathcal{R} f$.

2.2. Period-doubling renormalization. Despite its origins in the real setting, the renormalization operator actually "lives" in complex-analytic spaces. We need to introduce some notation before giving precise statements.

Let $g: U \rightarrow V$ be a quadratic-like map. This means that $g$ is a ramified holomorphic covering map of degree two, where $U$ and $V$ are simply-connected domains, where $U$ is compactly contained in $V, U \Subset V$. The annulus $V \backslash U$ is called the fundamental annulus of $g$. The filled-in Julia set of a quadratic-like map $g$ is the set

$$
K(g):=\bigcap_{n} g^{-n} V .
$$

Quadratic-like maps have two fixed points counted with multiplicity. If $K(g)$ is connected, then one of them, called $\alpha$, is either non-repelling or disconnecting (i.e., $K(g)$ becomes disconnected after removing $\alpha)$. The other fixed point is called $\beta$.

We say that $g$ is period-doubling renormalizable in Douady-Hubbard sense if there exist simply-connected sub-domains $U_{1}, V_{1}$ so that $g_{1}:=g^{2}: U_{1} \rightarrow V_{1}$ 
is also a quadratic-like map with connected filled-in Julia set and, furthermore, $K\left(g_{1}\right) \cap g\left(K\left(g_{1}\right)\right)$ is the $\beta$-fixed point of $g_{1}$. The renormalization in Douady-Hubbard sense is related to the period-doubling renormalization for unimodal maps in the following way: deep renormalizations of infinitely renormalizable real analytic unimodal maps have quadratic-like extensions which are infinitely renormalizable in Douady-Hubbard sense.

Two quadratic-like maps $h: U_{h} \rightarrow V_{h}$ and $g: U_{g} \rightarrow V_{g}$, both with connected filled-in Julia set, define the same quadratic-like germ if $K(h)$ coincides with $K(g)$ and $h$ coincides with $g$ in a neighborhood of $K(g)$. If $g$ is renormalizable, then the renormalization of the germ defined by $g$, denoted $\mathcal{R} g$, is the unique quadratic-like germ defined by the normalization of any possible induced map $g^{2}: U_{1} \rightarrow V_{1}$ which is a quadratic-like map with connected filled-in Julia set (normalize the germ using an affine conjugacy, setting the critical point at zero and the $\beta$-fixed point in $g_{1}$ to $1)$.

The operator $\mathcal{R}$ is called the period-doubling renormalization operator. In the setting of quadratic-like germs which have real values on the real line, there exists a unique fixed point to the period-doubling renormalization operator (proved by D. Sullivan; see also McM96]), denoted by $f^{\star}$. This fixed point satisfies the functional equation $\rho f^{\star}(z)=f^{\star} \circ f^{\star}(\rho z)$, for some $-1<\rho<0$ so that $f^{\star}(\rho)=\rho$. Indeed $\rho$ is the $\alpha$-fixed point of $f^{\star}$ or, what is the same, the $\beta$-fixed point of the quadratic-like restriction of the second iteration of $f^{\star}$ prior to its normalization to 1 in the definition of the renormalization operator above.

Given an analytic function $f: U \rightarrow \mathbb{C}$, where $U$ is an open set, define the open set

$$
\mathcal{D}_{U}^{n}(f):=\bigcap_{i=0}^{n-1} f^{-i} U .
$$

In other words, $\mathcal{D}_{U}^{n}(f)$ is the complex domain where $f^{n}$ is defined.

Recall that we defined the renormalization of a quadratic-like map as a quadraticlike germ. We would like to consider the renormalization operator as an operator acting on holomorphic functions, at least for those close enough to $f^{\star}$. To this end we need to know a little more about the fixed point $f^{\star}$ (see Lyu99): It is a consequence of the so-called a priori complex bounds [Su that we can choose simply-connected domains $U \Subset \tilde{U}$, and $N>0$ so that:

i. $f^{\star}$ has a continuous extension to $\bar{U}$ which is holomorphic in $U$.

ii. There exists an open set $V \Subset U$ with smooth boundary such that $f^{\star}: V \rightarrow$ $f^{\star} V$ is a quadratic-like map.

iii. We have that

$$
\rho^{N} \tilde{U} \Subset \mathcal{D}_{\left(f^{\star}\right)^{-1} V}^{2^{N}}\left(f^{\star}\right)
$$

in other words we can iterate $f^{\star}:\left(f^{\star}\right)^{-1} V \rightarrow V$ at least $2^{N}$ times on the domain $\rho^{N} \overline{\tilde{U}}$.

iv. Define $W:=\rho^{2 N} V$. Then $\left(f^{\star}\right)^{i} W$, for $i=0, \ldots, 2^{2 N}$, is disjoint from the annulus $\left.\rho^{N} V \backslash \rho^{N}\left(f^{\star}\right)^{-1} V\right|_{0}$.

Property iii implies that the quadratic-like germ of $\mathcal{R}^{N} f^{\star}$ can be obtained as a restriction of the $2^{N}$-th iteration of $f^{\star}: \bar{U} \rightarrow \mathbb{C}$ to the sub-domain $\rho^{N} \tilde{U}$ after normalizing it by the factor $\rho^{-N}$. So this normalized restriction of an iteration of $f^{\star}$ is defined on $\tilde{U}$, which contains the original domain of the definition of $f^{\star}: \bar{U} \rightarrow \mathbb{C}$. This argument is robust in the following way: for maps $f: \bar{U} \rightarrow \mathbb{C}$ close enough to 
$f^{\star}$, we can also use the same argument to prove that its $N$-th renormalization is defined at least on $\bar{U}$ as a normalized $2^{n}$-th iteration of $f$. This observation will be crucial to define the renormalization operator as an operator on an open set of a Banach space of holomorphic functions on $U$ which have a continuous extension to $\bar{U}$.

Property iv says that the first $2^{2 N}$ iterations by $f^{\star}$ of the fundamental annulus of the $2 N$-th renormalization of $f^{\star}$ does not intersect the fundamental annulus of the $N$-th renormalization of $f^{\star}$. This is a technical property used only in the proof of an auxiliary result (Lemma 4.1).

Denote by $\mathcal{B}(U)$ the Banach space of the complex analytic functions $g, D g(0)=$ 0 , with a continuous extension to $\bar{U}$, endowed with the sup norm, and by $\mathcal{B}_{\text {nor }}(U)$ denote the affine subspace of functions $g$ such that $g(1)=1$. Choose $\epsilon$ small enough such that for each $f$ in

$$
B\left(f^{\star}, \epsilon\right):=\left\{f \in \mathcal{B}_{\text {nor }}(U),\left|f-f^{\star}\right|_{\mathcal{B}(U)}<\epsilon\right\},
$$

the following holds:

- There exists an analytic continuation $\rho_{f}$ of the $\beta$-fixed point of the small Julia set associated with the $N$-th renormalization of $f^{\star}$. (At first glance a better notation could be $\beta_{\mathcal{R}^{N} f}$, but we note that a map $f$ close to $f^{\star}$ is not in general $N$ times renormalizable.)

- The map $f: f^{-1} V \rightarrow V$ is a quadratic-like map.

- We have $\rho_{f} \tilde{U} \Subset \mathcal{D}_{f^{-1} V}^{2^{N}}(f)$.

Such $\epsilon$ does exist due to properties i-iii above.

Define the complex analytic transformation $\tilde{\mathcal{R}}: B\left(f^{\star}, \epsilon\right) \rightarrow \mathcal{B}_{\text {nor }}(\tilde{U})$ as

$$
\tilde{\mathcal{R}} f(z):=\frac{1}{\rho_{f}} f^{2^{N}}\left(\rho_{f} z\right) .
$$

If $i: \mathcal{B}(\tilde{U}) \rightarrow \mathcal{B}(U)$ is the natural compact inclusion between these Banach spaces, define the complex analytic extension of the period-doubling operator as $\mathcal{R}:=i \circ \tilde{\mathcal{R}}$. This definition coincides with the previous definition for quadratic-like germs in the intersection of the domains of these operators. We should be careful here: maps in $B\left(f^{\star}, \epsilon\right)$, where the operator $\mathcal{R}$ is defined, are not necessarily renormalizable in the Douady-Hubbard sense. To avoid confusion, we will reserve the word "renormalizable" for quadratic-like maps (or germs) which are renormalizable in the Douady-Hubbard sense.

Two quadratic-like maps $g_{0}$ and $g_{1}$ are in the same hybrid class if there exists a quasiconformal conjugacy $\phi$ between them, in a neighborhood of their filled-in Julia sets, so that $\bar{\partial} \phi \equiv 0$ on $K\left(g_{0}\right)$. Note that quadratic-like maps in the hybrid class of $f^{\star}$ are infinitely renormalizable. Since there are no invariant line fields on the Julia set of $f^{\star}$, if there is a quasiconformal conjugacy between $f$ and $f^{\star}$, then $f$ belongs to the hybrid class of $f^{\star}$. It is well known $[\mathrm{Su}$ that unimodal maps which are infinitely period-doubling renormalizable have deep renormalizations with quadratic-like extensions which belong to the hybrid class of $f^{\star}$.

We are ready to make the precise statements. We are going to split the Main Theorem into two steps:

Theorem 1 (Exponential contraction: McM96 and Lyu99). There exists $\lambda<1$ so that, for every quadratic-like map $f$ which is in the hybrid class of $f^{\star}$, there 
exist $n_{0}=n_{0}(f)$ and $C=C(f)>0$ so that $\mathcal{R}^{n} f \in B\left(f^{\star}, \epsilon\right)$, for $n \geq n_{0}$, and $\left|\mathcal{R}^{n_{0}+n} f-f^{\star}\right|_{\mathcal{B}(U)} \leq C \lambda^{n}$.

The most attractive feature of this new proof is that it is essentially infinitesimal and has a "dynamical flavor": we will prove that the derivative of the renormalization operator is a contraction in the tangent space of the hybrid class (the contraction of the derivative of the renormalization operator on the hybrid class was proved by Lyubich Lyu99, but his proof is not infinitesimal). Moreover, the method seems to be as general as the previous ones: it also applies to the classical renormalization horseshoe Lyu99 and the Fibonacci renormalization operator Sm02a, for instance.

We will also obtain, as a corollary of the McMullen theory of towers [McM96], the local behavior of semi-attractive fixed points $[\mathrm{H}]$ and an easy application of the $\lambda$-lemma MSS that

Theorem 2 ([Lan, Lyu99). The period-doubling fixed point is hyperbolic, with codimension one stable manifold.

2.3. Parabolic domains for semi-attractive fixed points. Consider a complex Banach space $B$, and let $F: A \rightarrow B$ be a complex analytic operator defined in an open subset $A$ of $B$. Suppose that $p \in A$ is a fixed point for $F$. We say that $p$ is a semi-attractive fixed point for $F$ if:

- The value 1 is an eigenvalue for $D F_{p}$.

- There exists a Banach subspace $E^{s}$, with (complex) codimension one, which is invariant by the action of $D F_{p}$, and furthermore the spectrum of $D F_{p}$, restricted to $E^{s}$, is contained in $\{z:|z| \leq r\}$, where $r<1$.

The following result was proved by M. Hakim $[\mathrm{H}]$ for finite-dimensional complex Banach spaces $\left(\mathbb{C}^{n}\right)$, but the proof works just as well for a general complex Banach space:

Proposition $2.1([\mathrm{H}])$. Consider a compact complex analytic operator $F$, defined in an open set of a complex Banach space B. Let $p$ be a semi-attractive fixed point. Then one of the following statements holds:

(1) Curve of fixed points: There exists a complex analytic curve of fixed points which contains $p$.

(2) Parabolic domains (Petals): There exists $k \geq 1$ so that, for every $\epsilon>0$, there exists a connected open set $U$, which is forward invariant by the action of $F$ and whose diameter is smaller than $\epsilon$. Moreover,

$$
F^{n} u \rightarrow_{n} p, \text { for every } u \in U,
$$

where the speed of this convergence is subexponential: for each $u \in U$, there exists $C=C(u)$ so that

$$
\frac{1}{C} \frac{1}{n^{1 / k}} \leq\left|F^{n} u-p\right| \leq C \frac{1}{n^{1 / k}}
$$

An outline of Hakim's proof can be found in the Appendix.

\section{InFINITESIMAL CONTRACTION ON THE HORIZONTAL SPACE}

Let $f: V_{1} \rightarrow V_{2}$ be a quadratic-like map with connected Julia set and with an analytical extension to $\mathcal{B}_{\text {nor }}(U)$, with $K(f) \subset U$. The horizontal subspace (introduced by Lyubich Lyu99) of $f$, denoted $E_{f}^{h}$, is the subspace of the vectors 
$v \in \mathcal{B}(U)$ so that there exists a quasiconformal vector field in the Riemann sphere $\alpha$ satisfying $v=\alpha \circ f-D f \cdot \alpha$ in a neighborhood of $K(f)$, with $\bar{\partial} \alpha \equiv 0$ on $K(f)$ and $\alpha(0)=\alpha(1)=\alpha(\infty)=0$. We will not use the following information here, but certainly it will clarify the spirit of our methods: in an appropriated setting, the hybrid class is a complex analytic manifold, and the horizontal space is the tangent space of the hybrid class at $f$ (see Lyu99).

Proposition 3.1 ( $\mathrm{ALdM}])$. Let $f$ be a quadratic-like map with an extension to $\mathcal{B}_{\text {nor }}(U)$ and connected Julia set contained in $U$. Let $V \Subset U$ be a simply-connected domain with smooth boundary so that $f: V \rightarrow f(V)$ is a quadratic-like map with connected Julia set. Then there exist $C, \epsilon>0$ so that, if $|f-g|_{\mathcal{B}(U)} \leq \epsilon$ and $g: g^{-1} V \rightarrow V$ is a quadratic-like map with connected Julia set and no invariant line fields on $K(g)$, then, for every $v \in E_{g}^{h}$ there exists a $C|v|_{\mathcal{B}(U)}$-quasiconformal vector field $\alpha$ in $\overline{\mathbb{C}}$ so that $v=\alpha \circ g-D g \cdot \alpha$ on $g^{-1} V$.

Any family of $C$-quasiconformal vector fields in $\overline{\mathbb{C}}$ which vanishes at three common points is relatively compact in the uniform topology (any sequence has a subsequence which converges uniformly to a quasiconformal vector field). So the last result implies:

Corollary 3.2 ([ALdM] $)$. Assume that $\left(f_{n}, v_{n}\right) \rightarrow_{n}\left(f_{\infty}, v_{\infty}\right)$ in $\mathcal{B}_{\text {nor }}(U) \times \mathcal{B}(U)$, where $f_{i}: f_{i}^{-1} V \rightarrow V, i \in \mathbb{N} \cup\{\infty\}$, are quadratic-like maps with connected filled-in Julia sets $K\left(f_{i}\right) \subset V \Subset U$. Furthermore, assume that $v_{n} \in E_{f_{n}}^{h}$, for $n \in \mathbb{N}$. If $f$ does not support invariant line fields in $K(f)$, then $v_{\infty} \in E_{f}^{h}$. In particular $E_{f}^{h}$ is closed.

The following result gives a description of the action of the derivative $D R_{f}$ on horizontal vector fields $v=\alpha \circ f-D f \cdot \alpha$ in terms of $\alpha$ :

Proposition 3.3. Let $V$ be the neighborhood of $K\left(f^{\star}\right)$ as in Section 2.2. If $f \in$ $\mathcal{B}_{\text {nor }}(U)$ is close enough to $f^{\star}$ and $v=\alpha \circ f-D f \cdot \alpha$ on $f^{-1} V$, where $v \in \mathcal{B}(U)$ and $\alpha$ is a quasiconformal vector field in the Riemann sphere normalized by $\alpha(0)=$ $\alpha(1)=\alpha(\infty)=0$, then

$$
D \mathcal{R}_{f} \cdot v=r(\alpha) \circ \mathcal{R} f-D(\mathcal{R} f) \cdot r(\alpha),
$$

on $\bar{U}$, where

$$
r(\alpha)(z):=\frac{1}{\rho_{f}} \alpha\left(\rho_{f} z\right)-\frac{1}{\rho_{f}} \alpha\left(\rho_{f}\right) \cdot z .
$$

In particular, if $f$ is renormalizable, then $D \mathcal{R}_{f}\left(E_{f}^{h}\right) \subset E_{\mathcal{R} f}^{h}$.

Proof. Let $v \in \mathcal{B}(U)$ be a vector field. Consider $f \in B\left(f^{\star}, \epsilon\right)$. Denote, for $z \in$ $\mathcal{D}_{f^{-1} V}^{i}(f)$,

$$
a_{i}(z):=\left.\frac{\partial}{\partial t}(f+t v)^{i}(z)\right|_{t=0} .
$$

It is easy to see that

$$
\begin{gathered}
a_{1}(z):=v(z), \\
a_{i}(x):=v\left(f^{i-1}(z)\right)+D f\left(f^{i-1}(z)\right) a_{i-1}(z) .
\end{gathered}
$$


Then (we replaced $\rho_{f}$ by $\rho$ to simplify the notation)

$$
\begin{gathered}
\left(D \mathcal{R}_{f} \cdot v\right)(z)=\left.\frac{\partial}{\partial t} \frac{1}{\rho_{f+t v}}(f+t v)^{2^{N}}\left(\rho_{f+t v} z\right)\right|_{t=0} \\
=\frac{-D \rho \cdot v}{\rho} \frac{1}{\rho} f^{2^{N}}(\rho z)+\frac{1}{\rho}\left(a_{2^{n}}(\rho z)+D f^{2^{N}}(\rho z)(D \rho \cdot v) z\right) .
\end{gathered}
$$

Note that the right-hand side makes sense for $z \in 1 / \rho_{f} \mathcal{D}_{f^{-1} V}^{2^{N}}(f)$, in particular on $\bar{U}$. Since $v$ is equal to $\alpha \circ f-D f \cdot \alpha$ on $f^{-1} V$, by the above recursive expression for $a_{i}$ and an inductive argument we obtain that, for $i>1$,

$$
a_{i}=\alpha \circ f^{i}-D f^{i} \cdot \alpha \text { on } \mathcal{D}_{f^{-1} V}^{i}(f) .
$$

It follows that $D \mathcal{R} \cdot v$ can be rewritten as

$$
\begin{gathered}
\left(D \mathcal{R}_{f} \cdot v\right)(z) r \\
=\frac{-D \rho_{f} \cdot v}{\rho} \frac{1}{\rho} f^{2^{N}}(\rho z)+\frac{1}{\rho}\left(\alpha \circ f^{2^{N}}(\rho z)-D f^{2^{N}}(\rho z) \cdot \alpha(\rho z)\right. \\
\left.\quad+D f^{2^{N}}(\rho z)(D \rho \cdot v) z\right) \\
=\frac{-D \rho \cdot v}{\rho} \mathcal{R} f(z)+\frac{1}{\rho} \alpha(\rho \mathcal{R} f(z))-D(\mathcal{R} f)(z) \cdot \frac{1}{\rho} \alpha(\rho z)+D(\mathcal{R} f)(z) \cdot \frac{D \rho \cdot v}{\rho} z,
\end{gathered}
$$

so $D \mathcal{R}_{f} \cdot v=r(\alpha) \circ \mathcal{R} f-D(\mathcal{R} f) \cdot r(\alpha)$ on $\bar{U}$, where

$$
r(\alpha)(z):=\frac{1}{\rho_{f}} \alpha\left(\rho_{f} z\right)-\frac{D \rho_{f} \cdot v}{\rho_{f}} z .
$$

Since $\rho_{f}$ is the analytic continuation of a periodic point of $f$ with period $2^{N}$, we have $f^{2^{N}}\left(\rho_{f}\right)=\rho_{f}$, so

$D \rho_{f} \cdot v=a_{2^{N}}\left(\rho_{f}\right)+D f^{2^{N}}\left(\rho_{f}\right) D \rho_{f} \cdot v=\alpha\left(\rho_{f}\right)-D f^{2^{N}}\left(\rho_{f}\right) \alpha\left(\rho_{f}\right)+D f^{2^{N}}\left(\rho_{f}\right) D \rho_{f} \cdot v$,

hence $D \rho_{f} \cdot v=\alpha\left(\rho_{f}\right)$ and

$$
r(\alpha)(z):=\frac{1}{\rho_{f}} \alpha\left(\rho_{f} z\right)-\frac{1}{\rho_{f}} \alpha\left(\rho_{f}\right) z .
$$

Note that $r(\alpha)(0)=r(\alpha)(1)=r(\alpha)(\infty)=0$. Now the invariance of the horizontal subspace is an immediate consequence of Proposition 3.1 and Corollary 3.2 .

Note that, apart from the normalization by a linear vector field, $r(\alpha)$ is just the pullback of the vector field $\alpha$ by a linear map. In particular, if $\alpha$ is a $C$ quasiconformal vector field, then $r(\alpha)$ is also a $C$-quasiconformal vector field: this will be a key point in the proof of the infinitesimal contraction of the renormalization operator in the horizontal subspace (Proposition 3.9).

Let $f^{\star}: V_{1} \rightarrow V_{2}$ be a quadratic-like representative of the fixed point. The perioddoubling tower is the indexed family of quadratic-like maps $f_{i}^{\star}: \rho_{f^{\star}}^{-i} V_{1} \rightarrow \rho_{f^{\star}}^{-i} V_{2}$, $i \in \mathbb{N}$, defined by $f_{i}^{\star}(z):=\rho_{f^{\star}}^{-i} f^{\star}\left(\rho_{f^{\star}}^{i} \cdot z\right)$.

Proposition 3.4 ([McM96]). The period-doubling tower does not support invariant line fields: this means that there is not a measurable line field which is invariant by all (or even an infinite number of) maps in the period-doubling tower. 
Proposition 3.5 ([Su] and McM96]). Let $f$ be a quadratic-like map which admits a hybrid conjugacy $\phi$ with $f^{\star}$. Then

$$
\phi_{n}(z):=\rho_{f^{\star}}^{-n} \cdot \phi\left(\rho_{\mathcal{R}^{n-1} f} \cdots \rho_{f} \cdot z\right)
$$

converges to identity uniformly on compact sets in the complex plane. In particular, there exists $n_{0}=n_{0}(f)$ so that $\mathcal{R}^{n} f \in B\left(f^{\star}, \epsilon\right)$, for $n>n_{0}$, and $\mathcal{R}^{n} f \rightarrow_{n} f^{\star}$ on $\mathcal{B}_{\text {nor }}(U)$.

Theorem 1 says that this convergence is, indeed, exponentially fast. The following proposition has an easy proof:

Proposition 3.6. Let $\mathcal{R}=i \circ \tilde{\mathcal{R}}$, where $\tilde{\mathcal{R}}: \mathcal{V} \rightarrow \tilde{\mathcal{B}}$ is a $C^{1}$ operator defined in an open subset $\mathcal{V}$ of a Banach space $\mathcal{B}$ to another Banach space $\tilde{\mathcal{B}}$, and $i: \tilde{\mathcal{B}} \rightarrow \mathcal{B}$ is a compact linear transformation. Let $S \subset \mathcal{B} \times \mathcal{B}$ be a set with the following properties:

(1) Vector bundle structure: If $\left(f, v_{1}\right)$ and $\left(f, v_{2}\right) \in S$, then $\left(f, \alpha \cdot v_{1}+v_{2}\right) \in$ $S$, for every $\alpha \in \mathbb{C}$.

(2) Semicontinuity: If $\left(f_{n}, v_{n}\right) \rightarrow(f, v)$ and $\left(f_{n}, v_{n}\right) \in S$, then $(f, v) \in S$.

(3) Invariance: If $(f, v) \in S$, then $\left(\mathcal{R} f, D \mathcal{R}_{f} \cdot v\right) \in S$.

(4) Compactness: $\left\{\left(\tilde{\mathcal{R}} f, D \tilde{\mathcal{R}}_{f} \cdot v\right):(f, v) \in S,|v| \leq 1\right\}$ is a bounded set in $\tilde{\mathcal{B}} \times \tilde{\mathcal{B}}$.

(5) Uniform continuity: Denote $E_{f}:=\{(f, v):(f, v) \in S\}$. There exists $C>0$ so that, for every $f$ and $n \geq 0,\left|D \mathcal{R}_{f}^{n}\right|_{E_{f}} \leq C$.

(6) If $(f, v) \in S$, then $\left|D \mathcal{R}_{f}^{n} \cdot v\right| \rightarrow_{n} 0$.

Then for all $\lambda<1$, there is $N \in \mathbb{N}$ so that $\left|D \mathcal{R}_{f}^{N}\right|_{E_{f}} \leq \lambda$, for every $f$ so that $E_{f} \neq \varnothing$.

Proof. Since $\left\{\left(\tilde{\mathcal{R}} f, D \tilde{\mathcal{R}}_{f} \cdot v\right):(f, v) \in S,|v| \leq 1\right\}$ is a bounded set in $\tilde{\mathcal{B}} \times \tilde{\mathcal{B}}$, and $i: \tilde{\mathcal{B}} \rightarrow \mathcal{B}$ is a compact linear transformation, we have that

$$
K=\overline{\left\{\left(\mathcal{R} f, D \mathcal{R}_{f} \cdot v\right):(f, v) \in S,|v| \leq 1\right\}}
$$

is compact in $\mathcal{B}$. By the invariance of $S$ and the Semicontinuity property, $K \subset S$. We claim that, given a positive $\lambda<1$, then for every $(f, v) \in K$, there exist an open set in $K$

$$
B_{(f, v)}:=\left\{(g, w) \in K \text { satisfying }|g-f|<\epsilon_{(f, v)},|w-v|<\delta_{(f, v)}\right\}
$$

and $N_{(f, v)} \in \mathbb{N}$ so that $\left|D \mathcal{R}_{g}^{N} \cdot w\right|<\lambda$, for every $(g, w) \in B_{(f, v)}$ and $N \geq N_{(f, v)}$. Indeed, due to property (6), for each $(f, v) \in K$, there exists $N_{(f, v)} \in \mathbb{N}$ such that

$$
\left|D \mathcal{R}_{f}^{N} \cdot v\right| \leq \lambda / 3 C
$$

for all $N \geq N(f, v)$. Because $\mathcal{R}$ is $C^{1}$, we can choose $\epsilon_{(f, v)}$ so that if $|f-g|<\epsilon_{(f, v)}$, then

$$
\left|D \mathcal{R}_{f}^{N(f, v)}-D \mathcal{R}_{g}^{N(f, v)}\right|_{\mathcal{B}}<\lambda / 3 C^{2} .
$$

Suppose that $(g, w) \in K$, with $|f-g| \leq \epsilon$ and $|v-w| \leq \delta_{(f, v)}:=\lambda / 3 C M$, where $M:=\left|D \mathcal{R}_{f}^{N_{(f, v)}}\right|_{\mathcal{B}}$. Then, using the uniform continuity property, for $N \geq N_{(f, v)}$ we 
have (note that $|v|,|w| \leq C$ )

$$
\begin{aligned}
\left|D \mathcal{R}_{g}^{N} \cdot w\right| \leq & C\left|D \mathcal{R}_{g}^{N_{(f, v)}} \cdot w\right| \\
\leq & C\left|D \mathcal{R}_{g}^{N_{(f, v)}} \cdot w-D \mathcal{R}_{f}^{N_{(f, v)}} \cdot w\right| \\
& +C\left|D \mathcal{R}_{f}^{N_{(f, v)}} \cdot w-D \mathcal{R}_{f}^{N_{(f, v)}} \cdot v\right|+C\left|D \mathcal{R}_{f}^{N_{(f, v)}} \cdot v\right| \\
\leq & \lambda .
\end{aligned}
$$

Since $K$ is compact, we can choose a finite open covering for $K$, say $B_{\left(f_{i}, v_{i}\right)}, i=$ $1, \ldots, L$. Take $N=1+\max _{i} N_{\left(f_{i}, v_{i}\right)}$. This completes the proof.

Proposition 3.6 is a generalization of the following fact about compact linear operators $T: \mathcal{B} \rightarrow \mathcal{B}$ : if $T^{n} v \rightarrow 0$, for every $v \in \mathcal{B}$, then the spectral radius of $T$ is strictly smaller than one.

Let $\epsilon>0, U$ and $V$ as defined in Section 2.2. Recall that $K\left(f^{\star}\right) \subset V \Subset U$. Given $K>0$, denote by $\mathcal{A}(\epsilon / 2, K, V)$ the set of maps $f \in \mathcal{B}_{\text {nor }}(U)$ so that there exists a $K$-quasiconformal map $\phi$ in the complex plane so that $\phi(V) \subset \bar{U}$ and $\phi \circ f^{\star}=f \circ \phi$ on $V$; moreover, for $n \geq 0$, we have $\left|\mathcal{R}^{n} f-f^{\star}\right|_{\mathcal{B}(U)} \leq \epsilon / 2$. Note that $\mathcal{A}(\epsilon / 2, K, V)$ is closed.

Lemma 3.7. Let $\tilde{V}$ be a simply-connected set with smooth boundary so that $V \Subset$ $\tilde{V} \Subset f^{\star} V$. For $K$ small enough, the following holds: if $f$ belongs to the hybrid class of $f^{\star}$ and there exists a $K$-quasiconformal map $\phi: \mathbb{C} \rightarrow \mathbb{C}$ so that $\phi \circ f^{\star}=$ $f \circ \phi$ on $\left.\left(f^{\star}\right)^{-1} \tilde{V}\right|_{0}$, then the germ $\mathcal{R}^{i} f$ has a representative in $\mathcal{B}(U)$ and $\mathcal{R}^{i} f \in$ $\mathcal{A}(\epsilon / 2, K, V)$, for all $i \geq 1$.

Proof. Let $M$ be a constant so that $\left|f^{\star}\left(z_{1}\right)-f^{\star}\left(z_{2}\right)\right| \leq M\left|z_{1}-z_{2}\right|$, for all $z_{1}, z_{2} \in$ $\tilde{U}$. Choose $K$ small enough such that, for all $K$-quasiconformal map $\phi: \mathbb{C} \rightarrow \mathbb{C}$ satisfying $\phi(0)=0, \phi(1)=1$ and $\phi(\infty)=\infty$, we have

- $\max _{z \in \tilde{U} \cup f^{\star}(\tilde{U})}\left\{|\phi(z)-z|,\left|\phi^{-1}(z)-z\right|\right\} \leq \epsilon / 4 M$,

- $\bar{U} \subset \phi(\tilde{U})$,

- $\bar{U} \subset \phi^{-1}(\tilde{U})$ and

- $\phi(V) \subset \bar{U}$.

We are going to prove, by induction, that $\mathcal{R}^{i} f \in \mathcal{B}(U),\left|\mathcal{R}^{i} f-f^{\star}\right| \leq \epsilon$ and that there exists a $K$-quasiconformal map $\phi_{i}: \mathbb{C} \rightarrow \mathbb{C}$ such that $\phi_{i} \circ f^{\star}=\mathcal{R}^{i} f \circ \phi_{i}$ on $\bar{U}$. Since $\phi_{i}(V) \subset \bar{U}$, we will get $\mathcal{R}^{i} f \in \mathcal{A}(\epsilon, K, V)$.

For $i=1$, note that the assumptions imply that

$$
\phi_{1} \circ f^{\star}=\mathcal{R} f \circ \phi_{1} \quad \text { on } \frac{1}{\rho_{f^{\star}}} \mathcal{D}_{\left.\left(f^{\star}\right)^{-1} V\right|_{0}}^{2^{N}}\left(f^{\star}\right),
$$

which contains $\tilde{U}$. Here

$$
\phi_{1}(z):=\frac{1}{\rho_{f}} \phi\left(\rho_{f \star} z\right) .
$$

By our choice of $K, \bar{U} \subset \phi_{1}(\tilde{U})$, so $\mathcal{R} f$ has a representative in $\mathcal{B}(U)$, and for $z \in \bar{U}$,

$$
\begin{gathered}
\left|\mathcal{R} f(z)-f^{\star}(z)\right|=\left|\phi_{1} \circ f^{\star} \circ \phi_{1}^{-1}(z)-f^{\star}(z)\right| \\
\leq\left|\phi_{1} \circ f^{\star} \circ \phi_{1}^{-1}(z)-f^{\star} \circ \phi_{1}^{-1}(z)\right|+\left|f^{\star} \circ \phi_{1}^{-1}(z)-f^{\star}(z)\right| \leq \frac{\epsilon}{2} .
\end{gathered}
$$

Assume the inductive hypothesis for $i=n$. Then

$$
\phi_{n+1} \circ f^{\star}=\mathcal{R}^{n+1} f \circ \phi_{n+1} \quad \text { on } \quad \frac{1}{\rho_{f^{\star}}} \mathcal{D}_{\left(f^{\star}\right)^{-1} U}^{2^{N}}\left(f^{\star}\right),
$$


which contains $\tilde{U}$. Here

$$
\phi_{n+1}(z):=\frac{1}{\rho_{\mathcal{R}^{n} f}} \phi_{n}\left(\rho_{f^{\star}} z\right) .
$$

As in the case $i=1$, our choice of $K$ implies $\bar{U} \subset \phi_{n+1}(\tilde{U})$, so $\mathcal{R}^{n+1} f$ has a representative in $\mathcal{B}(U)$ and for $z \in \bar{U}$,

$$
\begin{gathered}
\left|\mathcal{R}^{n+1} f(z)-f^{\star}(z)\right|=\left|\phi_{n+1} \circ f^{\star} \circ \phi_{n+1}^{-1}(z)-f^{\star}(z)\right| \\
\leq\left|\phi_{n+1} \circ f^{\star} \circ \phi_{n+1}^{-1}(z)-f^{\star} \circ \phi_{n+1}^{-1}(z)\right|+\left|f^{\star} \circ \phi_{n+1}^{-1}(z)-f^{\star}(z)\right| \leq \frac{\epsilon}{2} .
\end{gathered}
$$

Lemma 3.8. For $K$ small enough the set $\mathcal{A}:=\mathcal{A}(\epsilon / 2, K, V)$ is invariant by the action of $\mathcal{R}$ and furthermore for every $g$ in the hybrid class of $f^{\star}$, there exists a complex analytic path $f_{t} \in \mathcal{A}$, for $|t| \leq 1$, and $N=N(g)$ such that $f_{1}:=\mathcal{R}^{N} g$ and $f_{0}=f^{\star}$.

Proof. Choose $K$ as in the proof of Lemma 3.7. We claim that $\mathcal{A}(\epsilon / 2, K, V)$ is invariant. Indeed, if $f \in \mathcal{A}$, then $\phi \circ f^{\star}=f \circ \phi$ on $V$, for some $K$-quasiconformal $\phi$ satisfying $\phi(0)=\phi(1)=\phi(\infty)$. But then

$$
\tilde{\phi}(z):=\frac{1}{\rho_{f}} \phi\left(\rho_{f^{\star}} z\right)
$$

satisfies

$$
\tilde{\phi} \circ f^{\star}=\mathcal{R} f \circ \tilde{\phi} \quad \text { on } \quad 1 / \rho_{f^{\star}} \mathcal{D}_{\left(f^{\star}\right)^{-1} V}^{2^{N}}\left(f^{\star}\right),
$$

which contains $V$. Furthermore $\tilde{\phi}$ is $K$-quasiconformal with the same normalization at the points 0,1 and $\infty$, so $\tilde{\phi}(V) \subset \bar{U}$. Of course

$$
\left|\mathcal{R}^{i} \mathcal{R} f-f^{\star}\right|_{\mathcal{B}(U)}=\left|\mathcal{R}^{i+1} f-f^{\star}\right|_{\mathcal{B}(U)} \leq \epsilon / 2,
$$

for $i \geq 0$. So $\mathcal{R} f \in \mathcal{A}$, proving that $\mathcal{A}$ is invariant.

Let $\tilde{V}$ be a simply-connected domain with smooth boundary such that $V \Subset \tilde{V} \Subset$ $f^{\star} V$. Choose $\delta>0$ such that if $f \in B\left(f^{\star}, \delta\right)$, then $f:\left.f^{-1} \tilde{V}\right|_{0} \rightarrow \tilde{V}$ is a quadraticlike map, where $\left.\tilde{V} \backslash f^{-1} \tilde{V}\right|_{0}$ is a holomorphic moving annulus. Using an argument similar to the proof of Lemma 2.2 in [Lyu02, we can reduce $\delta$, if necessary, such that for every $f \in B\left(f^{\star}, \delta\right)$ in the hybrid class of $f^{\star}$ there exists a $K$-quasiconformal map $\phi: \mathbb{C} \rightarrow \mathbb{C}$ such that $\phi \circ f^{\star}=f \circ \phi$ on $\left.\left(f^{\star}\right)^{-1} \tilde{V}\right|_{0}$.

Consider the following Beltrami path $f_{t}$ between $f$ and $f^{\star}$, induced by $\phi$ : if $\phi_{t}$, $|t| \leq 1$, is the unique normalized quasiconformal map such that $\bar{\partial} / \partial \phi_{t}=t \cdot \bar{\partial} / \partial \phi$, define $f_{t}=\phi_{t} \circ f^{\star} \circ \phi_{t}^{-1}$ on $\left.\phi_{t}\left(f^{\star}\right)^{-1} \tilde{V}\right|_{0}$. So $\phi_{t} \circ f^{\star}=f \circ \phi_{t}$ on $\left.\left(f^{\star}\right)^{-1} \tilde{V}\right|_{0}$. By Lemma 3.7. $\mathcal{R}^{i} f_{t} \in \mathcal{A}$, for $|t| \leq 1$. It is easy to check that $\mathcal{R} f_{t}$ is a complex analytic path.

By the topological convergence, for all $g$ in the hybrid class of $f^{\star}$ there exists $N(g)$ such that $\mathcal{R}^{N(g)} g \in B\left(f^{\star}, \delta\right)$. This finishes the proof.

We are ready to prove:

Proposition 3.9 (Infinitesimal contraction; cf. Lyu99). There exist $\lambda<1$ and $N>0$ such that $\left|D \mathcal{R}_{f}^{N}\right|_{E_{f}^{h}} \leq \lambda$, for every $f \in \mathcal{A}(\epsilon / 2, K, V)$. 
Proof. Consider the set $S:=\left\{(f, v): f \in \mathcal{A}, v \in E_{f}^{h}\right\}$. It is sufficient to verify the properties in the statement of Proposition 3.6. Since $\mathcal{A}$ is closed, property 2 follows from Corollary 3.2. Since $\mathcal{A}$ is invariant by $\mathcal{R}$, property 3 follows from Proposition 3.3. The compactness property is obvious, if $\epsilon$ is small enough. To prove the uniform continuity property, by Propositions 3.1 and 3.3 , we have that, for $(f, v) \in S$ and $n \geq 1$,

$$
D \mathcal{R}_{f}^{n} \cdot v=\alpha_{n} \circ \mathcal{R}^{n} f-D\left(\mathcal{R}^{n} f\right) \cdot \alpha_{n}
$$

on $\bar{U}$, with

$$
\alpha_{n}(z):=\frac{1}{\rho_{n-1} \ldots \rho_{0}} \alpha\left(\rho_{n-1} \ldots \rho_{0} z\right)-\frac{1}{\rho_{n-1} \cdots \rho_{0}} \alpha\left(\rho_{n-1} \ldots \rho_{0}\right) z,
$$

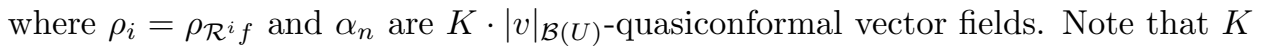
does not depend on $(f, v) \in S$ or $n \geq 1$. By the compactness of $K$-quasiconformal vector fields (recall that $\alpha_{n}(0)=\alpha_{n}(1)=\alpha_{n}(\infty)=0$ ), we get $\left|\alpha_{n}(z)\right| \leq M$ on the disk with center 0 and radius $1+\epsilon+\operatorname{diam} f^{\star}(U)+\operatorname{diam} U$, where $M$ depends only on $K$. Since $\left|\mathcal{R}^{n} f-f^{\star}\right| \leq \epsilon / 2$, for $n \geq 0$, there exists $C$, which depends only on $\epsilon$, so that $\left|\mathcal{R}^{n} f(z)\right|$ and $\left|D \mathcal{R}^{n} f(z)\right|$ are bounded by $C$, for $z \in \bar{U}$. So, if $v \in E_{f}^{h}$, we have that

$$
\begin{aligned}
\left|D \mathcal{R}_{f}^{n} \cdot v\right|_{\mathcal{B}(U)} \leq \mid & \left.\alpha_{n} \circ \mathcal{R}^{n} f\right|_{\mathcal{B}(U)}+\left|D\left(\mathcal{R}^{n} f\right) \cdot \alpha_{n}\right|_{\mathcal{B}(U)} \\
\leq & 2 M C|v|_{\mathcal{B}(U)}
\end{aligned}
$$

so $\left|D \mathcal{R}_{f}^{n}\right|_{E_{f}^{h}} \leq 2 M C$.

We are going to prove assumption 6. By Eq. (3), it is enough to show that $\alpha_{n} \rightarrow_{n} 0$ uniformly on $\overline{\mathbb{C}}$. Due the precompactness of the family $\alpha_{n}$, it is sufficient to prove that any convergent subsequence converges to zero.

Suppose $\alpha_{n_{k}} \rightarrow \alpha_{\infty}$. Define, for $1 \leq i \leq n$, the maps

$$
g_{i, n} \in \mathcal{B}_{\text {nor }}\left(\frac{1}{\rho_{n-1} \ldots \rho_{n-i}} \bar{U}\right)
$$

by

$$
g_{i, n}(z):=\frac{1}{\rho_{n-1} \ldots \rho_{n-i}} \mathcal{R}^{n-i} f\left(\rho_{n-1} \ldots \rho_{n-i} z\right) .
$$

We claim that $\bar{\partial} \alpha_{n}$ is an invariant Beltrami field on the finite tower $g_{1, n}, g_{2, n}, \ldots$, $g_{n, n}$. Indeed, define the $K$-quasiconformal vector field

$$
\eta_{i, n}(z):=\frac{1}{\rho_{n-1} \ldots \rho_{0}} \alpha\left(\rho_{n-1} \ldots \rho_{0} z\right)-\frac{1}{\rho_{n-i-1} \cdots \rho_{0}} \alpha\left(\rho_{n-i-1} \ldots \rho_{0}\right) z .
$$

Note that

$$
\eta_{i, n}(0)=\eta_{i, n}(\infty)=\eta_{i, n}\left(\frac{1}{\rho_{n-1} \cdots \rho_{n-i}}\right)=0
$$

Eq. (3) implies

$$
v_{i, n}=\eta_{i, n} \circ g_{i, n}-D g_{i, n} \cdot \eta_{i, n}
$$

on $\rho_{n-1}^{-1} \ldots \rho_{n-i}^{-1} \bar{U}$, where $v_{i, n}$ is a holomorphic vector field. So $\bar{\partial} \eta_{i, n}=\bar{\partial} \alpha_{n}$ is an invariant Beltrami field for $g_{i, n}$. This completes the proof of the claim.

By the topological convergence $\rho_{n-1} \cdots \rho_{n-i} \rightarrow_{n} \rho_{f^{*}}^{i}$, so for $n$ large, we have that $g_{i, n}$ and $v_{i, n}$ belong to $\mathcal{B}\left(\rho_{f^{\star}}^{-i} V\right)$. The topological convergence (Proposition 3.5) also implies $g_{i, n} \rightarrow_{n} f_{i}^{\star}$. Due to Eq. (4), if we fix $i, \eta_{i, n}$ is a relatively compact family 
of $K$-quasiconformal vector fields. By the usual diagonalization method, we can choose a subsequence of $n_{k}$, if necessary, such that there exists a quasiconformal vector field $\omega_{i}$, satisfying $\eta_{i, n_{k}} \rightarrow_{k} \omega_{i}$ uniformly on compact sets, for all $i$. Eq. (5) implies

$$
w_{i}=\omega_{i} \circ f_{i}^{\star}-D f_{i}^{\star} \cdot \omega_{i}
$$

on $\rho_{f^{\star}}^{-i} V$. Here $w_{i}$ is a holomorphic vector field.

By definition $\eta_{i, n}=\alpha_{n}+\gamma_{i, n}$, where $\gamma_{i, n}$ is a conformal vector field on the Riemann sphere. Once $\alpha_{n_{k}} \rightarrow_{k} \alpha_{\infty}$ on $\overline{\mathbb{C}}$, we get that $\gamma_{i, n_{k}}$ also converges to a conformal vector field $\sigma_{i}$. So $\bar{\partial} \omega_{i}=\bar{\partial} \alpha_{\infty}+\bar{\partial} \sigma_{i}=\bar{\partial} \alpha_{\infty}$. Therefore, Eq. (6) implies that the Beltrami field $\bar{\partial} \alpha_{\infty}$ is invariant on the period-doubling tower. Because there is no existence of invariant line fields in the period-doubling tower (see Proposition [3.4), $\bar{\partial} \alpha_{\infty} \equiv 0$ everywhere and, since $\alpha_{\infty}$ vanishes at three points, $\alpha_{\infty} \equiv 0$.

Hence $\alpha_{n} \rightarrow 0$ uniformly on compact sets in the complex plane, so we get by Eq. (3) that $\mathcal{R}_{f}^{n} \cdot v \rightarrow 0$. (We saw that $\left|\mathcal{R}^{n} f\right|$ and $\left|D\left(\mathcal{R}^{n} f\right)\right|$ are uniformly bounded on $\bar{U}$, for $n \geq 1$.) This finishes the proof.

Proof of Theorem 1. Let $g$ be a quadratic-like map in the hybrid class of $f^{\star}$. By Lemma 3.7, there exist $N(g)$ such that $\mathcal{R}^{N(g)} g \in \mathcal{A}$ and a complex analytic path $f_{t} \in \mathcal{A},|t| \leq 1$, such that $f_{0}=f^{\star}$ and $f_{1}=\mathcal{R}^{N(g)} g$. We are going to prove that

$$
\left.\frac{d f_{t}}{d t}\right|_{t=t_{0}} \in E_{f_{t_{0}}}^{h}
$$

for $\left|t_{0}\right| \leq 1$. Recall that we choose $\epsilon$ so that $\left.V \backslash f^{-1} V\right|_{0}$ is a holomorphic moving annulus on $f \in B\left(f^{\star}, \epsilon\right)$. Consider the new parametrization $f_{t_{0}+t}$. Then $\left.V \backslash f_{t_{0}+t}^{-1} V\right|_{0}$ is a holomorphic moving annulus defined for $D_{t_{0}}:=\left\{t:|t| \leq 1+\rho-\left|t_{0}\right|\right\}$. By the usual arguments, we can extend this holomorphic motion $h_{t}: \mathbb{C} \rightarrow \mathbb{C}$, with $t \in D_{t_{0}}$, $h_{0}(z)=z$, so that

$$
f_{t_{0}+t} \circ h_{t}=h_{t} \circ f_{t_{0}}
$$

on $\left.f_{t_{0}}^{-1} V\right|_{0}$. Derive with respect to $t$ to obtain

$$
\frac{d f_{t_{0}+t}}{d t} \circ h_{t}+D f_{t_{0}+t} \circ h_{t} \cdot \frac{d h_{t}}{d t}=\frac{d h_{t}}{d t} \circ f_{t_{0}} .
$$

Define the quasiconformal vector field

$$
\alpha(z):=\frac{d h_{t}}{d t}(z)
$$

So for $t=0$ we have

$$
\left.\frac{d f_{t}}{d t}\right|_{t=t_{0}}=\alpha \circ f_{t_{0}}-D f_{t_{0}} \cdot \alpha
$$

on $\left.f_{t_{0}}^{-1} V\right|_{0}$, which implies Eq. (77).

So, by the infinitesimal contraction, there exists $N$ so that

$$
\left|D \mathcal{R}_{f}^{N} \cdot v\right| \leq \frac{1}{2}
$$

for every $f \in \mathcal{A}, v \in E_{f}^{h}$. Define $M:=\max _{|t| \leq 1}\left|d f_{t} / d t\right|$ and recall that $\left|D \mathcal{R}_{f}\right|_{E_{f}^{h}}$ is uniformly bounded by some constant $C$, for every $f \in \mathcal{A}$. If $n=N q+r$, where 
$0 \leq r<N$, we have

$$
\begin{gathered}
\left|\mathcal{R}^{n} f_{1}-f^{\star}\right|_{\mathcal{B}(U)} \leq \int_{0}^{1}\left|\frac{d \mathcal{R}^{n} f_{t}}{d t}\right| d t \leq \int_{0}^{1}\left|D \mathcal{R}_{f_{t}}^{n} \cdot \frac{d f_{t}}{d t}\right| d t \\
\leq \int_{0}^{1}\left|D \mathcal{R}_{f_{t}}^{n} \cdot \frac{d f_{t}}{d t}\right| d t \leq 2^{-q} C^{N} M \leq 2 C^{N} M 2^{-\frac{n}{N}},
\end{gathered}
$$

which finishes the proof.

Remark 3.10. The first step in the above proof of Theorem 1 to prove that $\alpha_{n} \rightarrow 0$ (in the proof of Proposition [3.9), must be compared with the proof of Lemma 9.12 in [McM96]. In C. McMullen's argument, additional considerations should be taken to arrive at exponential contraction; first, it is proved that quasiconformal deformations (as the quasiconformal vector field $\alpha$ in the definition of the horizontal vectors) are $C^{1+\theta}$-conformal at the critical point (Lemma 9.12 in [McM96] and the deepness of the critical point have key roles in this proof), and then it is necessary to integrate this result. In M. Lyubich's argument Lyu99, first it is proved that the hybrid class is a complex analytic manifold and then the topological convergence is converted in exponential contraction via Schwartz's Lemma.

\section{Hyperbolicity of the PERIOD-DOUbling FIXED POINT}

Let $f$ be a quadratic-like map which is renormalizable with period $k$ : there exists a domain $W$, which contains the critical point, so that $f^{k}: W \rightarrow f^{k} W$ is a quadratic-like map with connected filled-in Julia set $K$. The sets $K_{0}:=K$, $K_{1}:=f(K), \ldots, K_{k-1}:=f^{k-1} K$ are called the small Julia sets of period $k$. This renormalization is simple if for every $0 \leq i, j<k$, either $K_{i} \cap K_{j}=\emptyset$ or $K_{i} \cap K_{j}=$ $\{p\}$, where $p$ does not cut $K_{j}$ in more than one piece. In the last case $p$ must be the so-called $\beta$-fixed point on $K_{j}$.

If $f$ is infinitely renormalizable, we say that $f$ satisfies the unbranched complex bounds property for an increasing sequence of renormalizations $i_{j}$ if there exists $\mu>0$ so that $\mathcal{R}^{i_{j}} f$ has a quadratic-like extension $\mathcal{R}^{i_{j}} f: W_{j} \rightarrow \mathcal{R}^{i_{j}} f\left(W_{j}\right)$ satisfying

i. $\bmod \mathcal{R}^{i_{j}} f\left(W_{j}\right) \backslash W_{j}>\mu$, and

ii. $\left(\mathcal{R}^{i_{j}} f\left(W_{j}\right) \backslash W_{j}\right) \cap P(f)=\emptyset$, for all $j>0$.

Here $P(f):=\left\{f^{k}(0), k>0\right\}$. The following lemma was proven by Lyubich (see Lemma 5.8 in [Lyu99), but for sake of completeness we included a proof.

Lemma 4.1. There exists $\delta>0$ so that the following holds: let $f \in \mathcal{B}_{\text {nor }}(U)$ be a map so that $\mathcal{R}^{n} f$ is defined and $\left|\mathcal{R}^{n} f-f^{\star}\right| \leq \delta$ for all $n \geq 0$. Then $f$ has infinitely many simply renormalizations satisfying the unbranched complex bounds.

Proof. Recall that in Section 2.2 we choose $\epsilon$ so that if $W:=\left.\rho^{2 N}\left(f^{\star}\right)^{-1} V\right|_{0}$, then $\left(f^{\star}\right)^{i} W$, for $i=0, \ldots, 2^{2 N}$, is disjoint from the annulus

$$
\left.\rho^{N} V \backslash \rho^{N}\left(f^{\star}\right)^{-1} V\right|_{0}
$$

By the continuity of $\mathcal{R}$, if $\delta$ is small, then $f^{i} W_{f}$ is disjoint from $\left.\rho_{f} V \backslash \rho_{f}(\mathcal{R} f)^{-1} V\right|_{0}$, for $i=0, \ldots, 2^{2 N}$, where

$$
W_{f}:=\left.\rho_{\mathcal{R} f} \rho_{f}\left(\mathcal{R}^{2} f\right)^{-1} V\right|_{0} .
$$

To prove that $f$ is infinitely renormalizable, it is enough to prove that $f:\left.f^{-1} V\right|_{0} \rightarrow$ $V$ is a quadratic-like map with a connected filled-in Julia set. Once $\mathcal{R}^{i} f$ satisfies the same assumption as $f$, we will get that $f$ is infinitely renormalizable. 
Indeed, it is easy to prove by induction that

$$
\rho_{\mathcal{R}^{i-1} f} \ldots \rho_{f} \bar{U} \subset \mathcal{D}_{f^{-1} V}^{2^{i N}}(f)
$$

and

$$
\mathcal{R}^{i} f(z):=\frac{1}{\rho_{\mathcal{R}^{i-1} f} \ldots \rho_{f}} f^{2^{i N}}\left(\rho_{\mathcal{R}^{i-1} f} \ldots \rho_{f} z\right) \text { on } \bar{U} .
$$

So $f^{j}(0)$ is well defined for $j \geq 0$, hence $f:\left.f^{-1} V\right|_{0} \rightarrow V$ has a connected filled-in Julia set.

Define $W_{i}:=\left.\rho_{\mathcal{R}^{i-1} f} \ldots \rho_{f}\left(\mathcal{R}^{i} f\right)^{-1} V\right|_{0}$. Note that

$$
f^{2^{i N}}: W_{i} \rightarrow f^{2^{i N}}\left(W_{i}\right)
$$

is a quadratic-like map with connected Julia set. Moreover

$$
\left.\bmod f^{2^{i N}}\left(W_{i}\right) \backslash W_{i} \sim V \backslash\left(f^{\star}\right)^{-1} V\right|_{0}>0 .
$$

Denote by $P(i)$ the postcritical set of this quadratic-like map. To prove the unbranched complex bounds it is enough to check that

$$
P(i) \cap f^{2^{(i+1) N}} W_{i+1}=P(i+1),
$$

for every $i$. Since $f^{2^{(j+1) N}} W_{j+1} \subset f^{2^{j N}} W_{j}$, for $j \geq 0$, this implies

$$
P(0) \cap f^{2^{i N}} W_{i}=P(i) .
$$

Indeed, by our choice of $\delta$, for

$$
W_{\mathcal{R}^{i f}}:=\left.\rho_{\mathcal{R}^{i+1} f} \rho_{\mathcal{R}^{i} f}\left(\mathcal{R}^{i+2} f\right)^{-1} V\right|_{0},
$$

we have that

$$
\left.\left(\mathcal{R}^{i} f\right)^{j} W_{\mathcal{R}^{i f}} \cap \rho_{\mathcal{R}^{i} f} V \backslash \rho_{\mathcal{R}^{i f}}\left(\mathcal{R}^{i+1} f\right)^{-1} V\right|_{0}=\emptyset,
$$

for $j=0, \ldots, 2^{2 N}$. Due to Eq. (9), we have

$$
f^{j 2^{i N}} W_{i+2} \cap\left(f^{2^{(i+1) N}} W_{i+1} \backslash W_{i+1}\right)=\emptyset
$$

for $j=0, \ldots, 2^{2 N}$. Since $f^{2^{(i+2) N}}: W_{i+2} \rightarrow f^{2^{(i+2) N}} W_{i+2}$ has a connected Julia set,

$$
P(i) \subset \bigcup_{j=0}^{2 N} f^{j 2^{i N}} W_{i+2},
$$

so Eq. (11) gives $P(i) \cap f^{2^{(i+1) N}} W_{i+1} \subset W_{i+1}$. The forward invariance of $P(i)$ by the action of $f^{2^{(i+1) N}}$ implies $P(i) \cap f^{2^{(i+1) N}} W_{i+1}=P(i+1)$.

It remains to prove that these renormalizations are simple. Because $\mathcal{R}^{i} f$ satisfies the same assumptions as $f$, it is enough to prove that the renormalization with period $2^{N}$ is simple. Indeed, if $\delta$ is small, the $\beta$-fixed point ( $\alpha$-fixed point) of the Julia set of $f^{2^{N}}: W_{1} \rightarrow f^{2^{N}} W_{1}$ is the analytic continuation of the $\beta$-fixed point ( $\alpha$-fixed point) of the Julia set of $\left(f^{\star}\right)^{2^{N}}: \rho^{N} f^{-1} V \rightarrow \rho^{N} V$. The same happens with the other small Julia sets in the same level. So two small Julia sets for $f$ intersect in a $\beta$-fixed point if and only if the corresponding small Julia sets of $f^{\star}$ intersect in a $\beta$-fixed point. Since all renormalizations of $f^{\star}$ are simple, we have finished the proof. 
Proof of Theorem 2. First, we will prove that

$$
\sigma\left(D \mathcal{R}_{f^{\star}}^{2}\right) \cap \mathbb{S}^{1} \subset\{1\} .
$$

Indeed, if $D \mathcal{R}_{f^{\star} \cdot v}=\lambda v$, then the vector $\tilde{v}(z):=\overline{v(\bar{z})}$ is a solution to $D \mathcal{R}_{f^{\star}} \cdot \tilde{v}=\bar{\lambda} \tilde{v}$. So if $\lambda \in \mathbb{S}^{1} \backslash\{-1,1\}$, then $\operatorname{codim} E^{h}>1$, which is a contradiction ( $E^{h}$ has codimension one Lyu99. The same result can be proven in an easy way using the argument explained in section 12 of $\mathrm{Sm02 \textrm {a }}$ ). Indeed, we can prove, using the contraction on the horizontal direction and results of [Sm03, which uses only elementary methods, that $\sigma\left(D \mathcal{R}_{f^{\star}}\right) \cap \mathbb{S}^{1} \subset\{1\}$, but the proof is more involved.

Furthermore $\sigma\left(D \mathcal{R}_{f^{\star}}\right)$ is not contained in $\mathbb{D}$ (see Lyubich Lyu99. We can also use the results in Sm03 to prove this claim). So either $f^{\star}$ is a hyperbolic fixed point (with a one-dimensional expanding direction) or it is a semi-attractive fixed point, since by Proposition 3.9 the derivative of the renormalization operator at the fixed point is a contraction on the horizontal space, which has codimension one. Assuming $f^{\star}$ is semi-attractive, we will arrive at a contradiction. Indeed, by Proposition 2.1, one of the following statements holds:

Case i. There exists a connected open set of maps $\mathcal{U} \subset \mathcal{B}_{\text {nor }}(U)$, whose diameter can be taken small, which is forward invariant by the action of $\mathcal{R}^{2}$ and so that each map in $\mathcal{U}$ is attracted at a subexponential speed to the fixed point $f^{\star}$. Because the maps in $\mathcal{U}$ are very close to $f^{\star}$ and $\mathcal{U}$ is forward invariant, all the maps in $\mathcal{U}$ are infinitely renormalizable (Lemma 4.1). Therefore, their filled-in Julia sets have empty interior. Consider two maps $g, \tilde{g}$ in $\mathcal{U}$ which admit a complex analytic path $g: \mathbb{D} \rightarrow \mathcal{U}$ between them $\left(g_{0}=g\right.$ and, for some $\left.|\lambda|<1, \tilde{g}=g_{\lambda}\right)$. Moreover, provided $\mathcal{U}$ is small enough, if $V$ is as in Section 2.2, then we can define a holomorphic motion $h: \mathbb{D} \times \partial V \cup \partial g^{-1} V \rightarrow \mathbb{C}$ as

$$
\begin{gathered}
h(\lambda, z)=z, \text { for } z \in \partial V \text { and } \\
g_{\lambda}(h(\lambda, z))=g(z), \text { for } z \in \partial g^{-1} V,
\end{gathered}
$$

for each $|\lambda|<1$. We can extend this holomorphic motion to a holomorphic motion $h: \mathbb{D} \times V \backslash g^{-1} V \rightarrow \mathbb{C}$ so that $h\left(\lambda, V \backslash g^{-1} V\right)=V \backslash g_{\lambda}^{-1} V$ and $h(\lambda, z)=z$, for every $z$ outside $V$. As usual, we are going to extend this holomorphic motion to a holomorphic motion $h: \mathbb{D} \times \mathbb{C} \backslash K(g) \rightarrow \mathbb{C}$ in the following way: denote

$$
A_{n}^{\lambda}:=\overline{g_{\lambda}^{-n} V \backslash g_{\lambda}^{-(n+1)} V}
$$

and assume that we had defined $h_{\lambda}:=h(\lambda, \cdot)$ on $A_{n}^{0}$. Then define by induction $h_{\lambda}$ on $A_{n+1}^{0}$ as the unique map $\tilde{h}_{\lambda}$ so that $g_{\lambda} \circ \tilde{h}_{\lambda}=h_{\lambda} \circ g$ on $A_{n+1}^{0}$ and $\tilde{h}_{\lambda}=h_{\lambda}$ on $A_{n}^{0} \cap A_{n+1}^{0}$. Note that $\tilde{h}_{\lambda}$ exists, once $g_{\lambda}: g_{\lambda}^{-1} V \rightarrow V$ has a connected Julia set, so $g_{\lambda}: A_{n+1}^{\lambda} \rightarrow A_{n}^{\lambda}$ are unbranched coverings of degree two.

So we have defined a holomorphic motion $h$ on the everywhere dense set $\mathbb{C} \backslash K(g)$ which commutes with the dynamics: we have $g_{\lambda} \circ h_{\lambda}=h_{\lambda} \circ g$ on $V \backslash K(g)$. By the $\lambda$-lemma MSS, this holomorphic motion extends to the whole Riemann sphere, so all maps $g_{\lambda}$ are quasiconformally conjugate. Since there is a piecewise complex analytic path between any two maps in $\mathcal{U}$, we conclude that all maps in $\mathcal{U}$ are in the same quasiconformal class. Note that the above construction does not give any upper bound for the quasiconformality of the conjugacy: the quasiconformality could be large when the Kobayashi distance between $g$ and $\tilde{g}$ on $\mathcal{U}$ is large.

We claim that, provided $\mathcal{U}$ is small enough, it is possible to choose a quasiconformal conjugacy between any two maps in $\mathcal{U}$ so that the quasiconformality 
is uniformly bounded outside their filled-in Julia sets, using the argument in the proof of Lemma 2.3 in Lyu02: in a small neighborhood $\mathcal{V} \subset \mathcal{B}_{\text {nor }}(U)$ of $f^{\star}$, $g: g^{-1} V \rightarrow V$ is a quadratic-like restriction of $g$ (but note that the Julia sets of these quadratic-like restrictions are not, in general, connected). This defines the holomorphic moving fundamental annulus $V \backslash g^{-1} V$. In particular, provided $\mathcal{U}$ is small enough, there exists $B>0$ so that for every $g_{0}$ and $g_{1}$ which belongs to $\mathcal{U}$, there exists a $B$-quasiconformal mapping $h$ between $\mathbb{C} \backslash g_{0}^{-1} V$ and $\mathbb{C} \backslash g_{1}^{-1} V$ so that $h \equiv I d$ on $\mathbb{C} \backslash V$ and $g_{1} \circ h=h \circ g_{0}$ on $\partial g_{0}^{-1} V$. Since the Julia sets of $g_{0}$ and $g_{1}$ are connected, we can extend $h$, as in the previous paragraph, to a $B$-quasiconformal map

$$
h: \mathbb{C} \backslash K\left(g_{0}\right) \rightarrow \mathbb{C} \backslash K\left(g_{1}\right)
$$

which is a conjugacy on $g_{0}^{-1} V \backslash K\left(g_{0}\right)$. The advantage of this new conjugacy is that $B$ is uniform for every $g_{1}$ and $g_{0}$ in $\mathcal{U}$. Once by the previous paragraph we already know that $g_{0}$ and $g_{1}$ are in the same quasiconformal class and $h$ has a quasiconformal extension $h_{g_{0}, g_{1}}$ to $\mathbb{C}$ (this follows as in the proof of Lemma 1, in [DH, p. 302]: if $\tilde{h}$ is a quasiconformal conjugacy between $g_{0}$ and $g_{1}$, then $\tilde{h}^{-1} \circ h$ commutes with $g_{0}$ outside $K\left(g_{0}\right)$, which implies that $\tilde{h}^{-1} \circ h$ extends to a homeomorphism in $\mathbb{C}$ which coincides with $I d$ on $K\left(g_{0}\right)$. By the Rickmann removability theorem (see the statement in $[\overline{D H}]$ ), this map is a quasiconformal homeomorphism, so $h$ is a quasiconformal homeomorphism). This finishes the proof of the claim.

By Lemma 4.1, for every $g_{0} \in \mathcal{U}$, the quadratic-like map $g_{0}: g_{0}^{-1} V \rightarrow V$ has infinitely many simply renormalizations with unbranched complex bounds. In particular there are no invariant line fields supported on their filled-in Julia sets (Theorem 10.2 in [McM94]), and hence the quasiconformality of the conjugacy $h_{g_{0}, g_{1}}: \mathbb{C} \rightarrow \mathbb{C}$ is uniformly bounded on the whole complex plane by $B$.

But $f^{\star}$ is a boundary point of $\mathcal{U}$, so there exists a sequence $g_{n} \in \mathcal{U}$ so that $g_{n} \rightarrow_{n} f^{\star}$ on $\mathcal{B}(U)$. By the previous paragraph, for each $n$ there exists a $B$ quasiconformal map $h_{n}: \mathbb{C} \rightarrow \mathbb{C}$ so that

$$
g_{n} \circ h_{n}=h_{n} \circ g_{0} \text { on } g_{0}^{-1} V .
$$

Furthermore $h_{n}(0)=0, h_{n}(1)=1$ and $h_{n}(\infty)=\infty$. By the compactness of $B$ quasiconformal maps, we can assume that $h_{n} \rightarrow_{n} h$ uniformly on compact sets in $\mathbb{C}$, where $h$ is a $B$-quasiconformal map. Taking the limit in Eq. (13), we get $f^{\star} \circ h=h \circ g_{0}$ on $g_{0}^{-1} V$. Since there are no invariant line fields supported on the filled-in Julia set of $f^{\star}$, the map $h$ is a hybrid conjugacy between $f^{\star}$ and $g_{0}$. But this implies that the subexponential speed of convergence given by Proposition 2.1 is impossible, since by Theorem 1 the iterations of the renormalization operator of maps in the hybrid class of $f^{\star}$ converges to $f^{\star}$ exponentially fast.

Case ii. There exists a connected complex analytic curve of fixed points which contains $f$. This case can be ruled out by the Rigidity Theorem from Lyu97, but instead we will apply essentially the same argument used in Case i: Note that in a similar way we can prove that all these fixed points of the operator $\mathcal{R}^{2}$ are quadratic like maps which are infinitely renormalizable: in particular their filled-in Julia sets have empty interior. Use the $\lambda$-lemma [MSS] to conclude that all these fixed points are quasiconformaly conjugated (the argument is as in Case i). Since the fixed point $f^{\star}$ does not support invariant line fields in its filled-in Julia set, we conclude that all these fixed points are hybrid conjugated, which is impossible, 
since iterations of the renormalization operator of maps in the hybrid class of $f^{\star}$ converges to the fixed point $f^{\star}$.

So we have concluded that $f^{\star}$ must be a hyperbolic fixed point with codimension one stable manifold.

\section{Appendix A. Outline of HaKim's PRoof}

To convince the reader of the existence of parabolic petals for semi-attractive compact operators in Banach spaces, we will give an outline of Hakim's proof of the existence of parabolic domains: we do not claim any sort of originality for ourselves in the following exposition and we refer to the quite clear work $[\mathrm{H}$ for details. We will use the notation introduced in Section 2.3. Consider a complex analytic operator $T$ with a semi-attractive fixed point 0 . Assume $D T_{0} \cdot v=v, v \neq 0$. In the following lines, we will identify $B$ with $\mathbb{C} \times E^{s}$ by the isomorphism $(x, y) \rightarrow x \cdot v+y$.

By the Stable Manifold Theorem for compact operators (see Manẽ [M]), for $\delta>0$ and $\epsilon>0$ small the set

$$
W_{\delta, \epsilon}^{s}=\left\{x: \exists C \text { s.t. }\left|T^{n} x\right|<\delta \text { and }\left|T^{n} x\right| \leq C(1-\epsilon)^{n}, \text { for } n \geq 0\right\}
$$

is a codimension one complex analytic manifold. More precisely, there exists a holomorphic function $\psi: V \rightarrow \mathbb{C}$, where $V$ is a neighborhood of 0 on $E^{s}$, with $D \psi(0)=0$, so that

$$
W_{\delta, \epsilon}^{s}=\{(\psi(y), y): y \in V\} .
$$

In particular, after the local biholomorphic change of variables

$$
\begin{aligned}
& X=x+\psi(y), \\
& Y=y,
\end{aligned}
$$

it is possible to represent $T$ as $T: \mathbb{C} \times E^{s} \rightarrow \mathbb{C} \times E^{s}$, where $T(x, y)=\left(x^{\prime}, y^{\prime}\right)$, with

$$
\begin{aligned}
& x^{\prime}=F(x, y)=a_{1}(y) x+O_{y}\left(x^{2}\right), \\
& y^{\prime}=G(y)+x h(x, y),
\end{aligned}
$$

where $G$ is a (compact) contraction around 0 and $a_{1}(0)=1$. After the local biholomorphic change of variables

$$
\begin{aligned}
& X=v(y) x, \\
& Y=y,
\end{aligned}
$$

where

$$
v(y):=\prod_{i \geq 0} a_{1}\left(G^{i}(y)\right)
$$

we can assume that $a_{1} \equiv 1$.

Note that, for every $n, T$ has the form

$$
\begin{aligned}
& x^{\prime}=F(x, y)=x+\sum_{2 \leq i \leq n} a_{i}(y) x^{i}+O_{y}\left(x^{n+1}\right), \\
& y^{\prime}=G(y)+x h(x, y),
\end{aligned}
$$

where $G$ is a (compact) contraction around 0 . We claim that we can assume, after certain biholomorphic changes of variables, that $a_{2}, a_{3}, \ldots, a_{n}$ do not depend on 
$y$. Indeed, assume by induction that $T$ can be put in the form

$$
\begin{aligned}
& x^{\prime}=F(x, y)=x+\sum_{2 \leq i \leq n} \tilde{a}_{i} x^{i}+\tilde{a}_{n+1}(y) x^{n+1}+O_{y}\left(x^{n+2}\right), \\
& y^{\prime}=G(y)+x h(x, y) .
\end{aligned}
$$

Then after the local change of variables

$$
\begin{aligned}
& X=x+v(y) x^{n+1}, \\
& Y=y,
\end{aligned}
$$

where $v(y):=\sum_{i \geq 0}\left(\tilde{a}_{n+1}\left(G^{i}(y)\right)-\tilde{a}_{n+1}(0)\right), T$ will have the form

$$
\begin{aligned}
& x^{\prime}=F(x, y)=x+\sum_{2 \leq i \leq n} \tilde{a}_{i} x^{i}+\tilde{a}_{n+1}(0) x^{n+1}+\tilde{a}_{n+2}(y) x^{n+2}+O_{y}\left(x^{n+2}\right), \\
& y^{\prime}=G(y)+x h(x, y) .
\end{aligned}
$$

Now we are going to introduce the concept of multiplicity of the fixed point 0 for transformations on the form of Eq. (17). By the implicit function theorem, for each transformation in that form there exists a complex analytic curve $y: U \subset \mathbb{C} \rightarrow E^{s}$, with $0 \in U$ and $y(0)=0$, which is the unique solution for the equation

$$
y(x)=G(y(x))+x h(x, y(x)) .
$$

Consider the function $q: U \rightarrow \mathbb{C}$ defined by

$$
q(x):=F(x, y(x))-x .
$$

The multiplicity of $T$ at 0 is defined as the order of $q$ at 0 . Note that the multiplicity of $T$ at 0 is finite if and only if 0 is an isolated fixed point, and infinite if and only if $q(x)$ vanishes everywhere and $(x, y(x))$ is a complex analytic curve of fixed points for $T$ (which contains all the fixed points in a neighborhood of 0 ). Moreover, if $T$ has the form Eq. (18), with $\tilde{a}_{2}=\cdots=\tilde{a}_{n-1}=0$ and $\tilde{a}_{n} \neq 0$, then the multiplicity of $T$ is exactly $n$.

Consider a transformation $T$ as in Eq. (17) and a biholomorphic change of variables $W(x, y)=(X, Y)$ of the type

$$
\begin{aligned}
& X=x+v(y) x^{k}, \\
& Y=y,
\end{aligned}
$$

where $v$ is a holomorphic function and $k>1$. Then $W^{-1} \circ T \circ W$ has also the form in Eq. (17). Moreover

Proposition A.1. The multiplicity of $W^{-1} \circ T \circ W$ at 0 is equal to the multiplicity of $T$ at 0 .

Proof. (suggested by M. Lyubich) Assume that it is finite (otherwise the invariance is trivial): then 0 is an isolated fixed point. Consider the one-parameter family of change of variables $W_{\lambda}$ defined by

$$
\begin{aligned}
& X=x+\lambda v(y) x^{k}, \\
& Y=y .
\end{aligned}
$$

Then $W_{\lambda}^{-1} \circ T \circ W_{\lambda}$ has the form

$$
\begin{aligned}
x^{\prime} & =F_{\lambda}(x, y)=x+O_{y, \lambda}\left(x^{2}\right), \\
y^{\prime} & =G_{\lambda}(y)+x h_{\lambda}(x, y) .
\end{aligned}
$$


Note that we can choose $\delta_{0}$ small enough so that for all $|\lambda| \leq 1,0$ is the unique fixed point for $W_{\lambda}^{-1} \circ T \circ W_{\lambda}$ on $\left\{(x, y),|x| \leq \delta_{0},|y| \leq \delta_{0}\right\}$. Moreover, by the implicit function theorem and the compactness of $\{\lambda:|\lambda| \leq 1\}$ there exists a holomorphic function $y_{\lambda}(x)=y(\lambda, x)$, defined on

$$
\left\{\lambda:|\lambda|<1+\delta_{1}\right\} \times\left\{x:|x|<\delta_{2}\right\}
$$

so that

$$
y_{\lambda}(x)=G_{\lambda}(y)+x h_{\lambda}\left(x, y_{\lambda}(x)\right) .
$$

Choosing $\delta_{1}, \delta_{2}$ small enough, for each $\lambda$ the point 0 is the unique solution for the equation

$$
q_{\lambda}(x):=F_{\lambda}\left(x, y_{\lambda}(x)\right)-x=0
$$

on $\left\{x:|x| \leq \delta_{1}\right\}$. By Rouché's Theorem, ord $d_{0} q_{\lambda}$ does not depend on $\lambda$.

Assume that $T$ has the form of Eq. (17) and finite multiplicity $n$. After appropriated changes of variables, we can assume that $a_{2}, \ldots, a_{2 n-1}$ does not depend on $y$. Since the multiplicity is invariant by the above changes of variables, we conclude that $a_{2}=\cdots=a_{n-1}=0$ and $a_{n} \neq 0$. Doing appropriated changes of variables in the form of Eq. (21) (indeed, in this case $v$ does not depend on $y$ ) and replacing the variable $x$ by $\theta x$, for some $\theta \neq 0$, if necessary, it is possible to put $T$ in the form

$$
\begin{aligned}
x^{\prime} & =x-\frac{1}{n-1} x^{n-1}+a x^{2(n-1)}+O_{y}\left(|x|^{2(n-1)+1}\right), \\
y^{\prime} & =G(y)+x h(x, y) .
\end{aligned}
$$

Under the above form, the set

$$
P_{R, \rho}=\left\{(x, y):\left|x^{n-1}-\frac{1}{2 R}\right|<\frac{1}{2 R} \text { and }|y|<\rho\right\}
$$

is a parabolic domain, provided $R$ and $\rho$ are small enough. Here Hakim's proof is very similar to the one-dimensional situation: make the "change of variables"

$$
\begin{aligned}
& X=x^{n-1}, \\
& Y=y,
\end{aligned}
$$

and

$$
\begin{aligned}
& X=1 / x, \\
& Y=y,
\end{aligned}
$$

to put $T$ in the form

$$
\begin{aligned}
& x^{\prime}=x+1+c \frac{1}{x}+O_{y}\left(\frac{1}{|x|^{1+1 /(n-1)}}\right), \\
& y=G(y)+O_{y}\left(\frac{1}{|x|^{1 /(n-1)}}\right),
\end{aligned}
$$

and now the proof is easy.

\section{ACKNOWLEDGEMENTS}

I thank IMS-SUNY at Stony Brook and the University of Toronto, and especially M. Lyubich for my wonderful stay at Stony Brook and Toronto, where this work was done. I am also indebted to M. Lyubich for the useful comments and suggestions. Finally I sincerely thank my new home, ICMC-USP, where the final revision was done. 


\section{REFERENCES}

[AB] L. Ahlfors and L. Bers. Riemann's mapping theorem for variable metrics. Ann. of Math., 72:385-404, 1960. MR0115006 (22:5813)

[ALdM] A. Avila, M. Lyubich and W. de Melo. Regular or stochastic dynamics in real analytic families of unimodal maps. Invent. Math. 154:451-550, 2003. MR2018784

[DH] A. Douady and J. Hubbard. On the dynamics of polynomial-like mappings. Ann. Sci. École Norm. Sup (4), 18:287-343, 1985. MR0816367 (87f:58083)

[H] M. Hakim. Attracting domains for semi-attractive transformations of $C^{p}$. Publ. Mat. 38, 479-499, 1994. MR1316642 (96a:32050)

[Lan] O. Lanford. A computer-assisted proof of the Feigenbaum conjectures. Bull. Amer. Math. Soc. 6:427-434, 1982. MR0648529 (83g:58051)

[Lyu97] M. Lyubich. Dynamics of quadratic polynomials, I-II. Acta Math., 178:185-297, 1997. MR.1459261 (98e:58145)

[Lyu99] M. Lyubich. Feigenbaum-Coullet-Tresser universality and Milnor's hairness conjecture. Ann. of Math., 149:319-420, 1999. MR1689333(2000d:37051)

[Lyu02] M. Lyubich. Almost every real quadratic map is either regular or stochastic. Ann. of Math., 156:1-78, 2002. MR1935840 (2003i:37032)

[McM94] C. McMullen. Complex dynamics and renormalization. Annals of Mathematics Studies, 135, Princeton University Press, Princenton, 1994. MR1312365 (96b:58097)

[McM96] C. McMullen. Renormalization and 3-manifolds which fiber over the circle. Annals of Mathematics Studies, 142, Princeton University Press, Princenton, 1996. MR1401347 (97f:57022)

[M] R. Mañé. Lyapounov exponents and stable manifolds for compact transformations. in Geometric dynamics (Rio de Janeiro, 1981), Lectures Notes in Math. 1007, 522-57\%. MR0730286 (85j:58126)

[MSS] R. Mañé; P. Sad and D. Sullivan. On the dynamics of rational maps. Ann. Sci. Ecole Norm. Sup., 16:193-217, 1983. MR0732343 (85j:58089)

[Sm01] D. Smania. Renormalization theory for multimodal maps. Eletronic Preprint, IMPA, 2001. Avaliable at www.icmc.usp.br/ smania/.

[Sm02a] D. Smania. Puzzle geometry and rigidity: The Fibonacci cycle is hyperbolic. Eletronic Preprint, 2002. Avaliable at www.icmc.usp.br/ smania/.

[Sm03] D. Smania. On Feigenbaum eigenvectors. In preparation, 2003.

[Su] D. Sullivan. Bounds, quadratic differentials and renormalization conjectures. in American Mathematical Society Centennial publications: Vol. II (Providence, RI, 1988, 417-466, Amer. Math. Soc, Providence, 1992. MR1184622 (93k:58194)

Department of Mathematics, University of Toronto, Toronto, Ontario, Canada M5S 1A1

Current address: Departamento de Matemática, ICMC-USP-Campus de São Carlos, Caixa Postal 668, São Carlos-SP, CEP 13560-970, Brazil

E-mail address: smania@icmc.usp.br

$U R L$ : www.icmc.usp.br/ smania/ 